\title{
Diagnostic accuracy of 22/25-gauge core needle in endoscopic ultrasound-guided sampling: systematic review and meta-analysis
}

\author{
Hyoung-Chul Oh ${ }^{1,}$, , Hyun Kang ${ }^{2,},{ }^{\star}$, Jae Young Lee ${ }^{1}$, Geun Joo Choi ${ }^{2}$, and Jung Sik Choi ${ }^{3}$
}

${ }^{1}$ Division of Gastroenterology, Department of Internal Medicine, ${ }^{2}$ Department of Anesthesiology, Chung-Ang University College of Medicine, Seoul; ${ }^{3}$ Division of Gastroenterology, Department of Internal Medicine, Inje University Busan Paik Hospital, Busan, Korea

Received: March 10, 2016

Revised : June 13, 2016

Accepted: June 28, 2016

\section{Correspondence to}

Hyoung-Chul Oh, M.D.

Division of Gastroenterology,

Department of Internal Medicine, Chung-Ang University College of Medicine, 102 Heukseok-ro, Dongjak-gu, Seoul o6973, Korea

Tel: +82-2-6299-3149

Fax: $+82-2-6299-2040$

E-mail: ohcgi@cau.ac.kr

*These authors contributed equally to this work.
Background/Aims: To compare the diagnostic accuracy of endoscopic ultrasound-guided core needle aspiration with that of standard fine-needle aspiration by systematic review and meta-analysis.

Methods: Studies using 22/25-gauge core needles, irrespective of comparison with standard fine needles, were comprehensively reviewed. Pooled sensitivity, specificity, diagnostic odds ratio (DOR), and summary receiver operating characteristic curves for the diagnosis of malignancy were used to estimate the overall diagnostic efficiency.

Results: The pooled sensitivity, specificity, and DOR of the core needle for the diagnosis of malignancy were 0.88 ( $95 \%$ confidence interval [CI], 0.84 to 0.90 ), 0.99 ( $95 \%$ CI, 0.96 to 1), and 167.37 (95\% CI, 65.77 to 425.91 ), respectively. The pooled sensitivity, specificity, and DOR of the standard needle were 0.84 (95\% CI, 0.79 to 0.88 ), 1 (95\% CI, 0.97 to 1), and 130.14 (95\% CI, 34.00 to 495.35 ), respectively. The area under the curve of core and standard needle in the diagnosis of malignancy was 0.974 and 0.955 , respectively. The core and standard needle were comparable in terms of pancreatic malignancy diagnosis. There was no significant difference in procurement of optimal histologic cores between core and standard needles (risk ratio [RR], $0.545 ; 95 \% \mathrm{CI}, 0.187$ to 1.589). The number of needle passes for diagnosis was significantly lower with the core needle (standardized mean difference, $-0.72 ; 95 \%$ CI, -1.02 to -0.41 ). There were no significant differences in overall complications (RR, 1.26; $95 \%$ CI, 0.34 to 4.62) and technical failure (RR, 5.07; 95\% CI, o.68 to 37.64).

Conclusions: Core and standard needles were comparable in terms of diagnostic accuracy, technical performance, and safety profile.

Keywords: Endoscopic ultrasound; Fine needle aspiration; Core needle

\section{INTRODUCTION}

Endoscopic ultrasound-guided fine-needle aspiration (EUS-FNA) has become the main diagnostic procedure for pancreaticobiliary disorders, in particularly pancreatic cancer. Pooled sensitivity and specificity of EUS-
FNA have been reported to be $85 \%$ to $86.8 \%$ and $95.8 \%$ to $98 \%$, respectively $[1,2]$. Although EUS-FNA is an effective diagnostic tool for the determination of correct etiology for solid pancreatic masses, it has several limitations. Cytological investigation of the aspirated materials is only able to establish cellular diagnosis. Certain neo- 
plasms, such as lymphomas and gastrointestinal stromal tumor, are difficult to diagnose without histologic samples, because in these cases, knowledge of the tissue architecture and original arrangement are essential for accurate pathological diagnosis including immunohistochemical analysis [3,4].

Due to the intrinsic limitations of cytology sampling, EUS-Trucut biopsy with a 19-gauge (G) needle and $19 \mathrm{G}$ biopsy needle with reverse bevel technology were developed to enable the procurement of tissue core for histologic analysis $[5,6]$. However, some technical difficulties still remain when performing transduodenal passage. Recently, 22 and $25 \mathrm{G}$ fine needle biopsy devices with reverse bevel technology have become available to facilitate transduodenal sampling [7]. However, it is uncertain if novel core needle increases the diagnostic accuracy by obtaining sufficient amount of tissue. The aim of this study was to perform a systematic review and meta-analysis to determine the diagnostic accuracy of $22 / 25 \mathrm{G}$ EUS-guided core needle aspiration in comparison with that of standard FNA.

\section{METHODS}

\section{Search strategy}

Our study was performed according to the recommendations on the conduct and reporting of systematic reviews and meta-analyses outlined by the Preferred Reporting Items in Systematic Reviews and Meta-Analyses statement [8], the Meta-Analysis of Observational Studies in Epidemiology [9], and the Cochrane Diagnostic Test Accuracy Working Group [10]. We conducted a literature search of Ovid MEDLINE, EMBASE, and Cochrane Central Register of Controlled Trials (CENTRAL) from the earliest available date of indexing through to March 23, 2015.

The search terms included "Core needle," "Pro Core," "Procore," "Core biopsy, "Histology EUS needle," "EUS histology needle," "biopsy needles for EUS," and "side fenestration." The literature search included potential studies without language, publication date, or other restrictions. We also checked the reference lists of all included studies for additional references.

\section{Study selection}

Studies were included if they met the following criteria: (1) randomized clinical trial, cohort study, or case-control study; (2) clinical trial based on humans, rather than animals; (3) histologically proven diagnosis; (4) evaluated the accuracy, sensitivity or specificity of core needle; and (5) absolute numbers of true-positive (TP), false-positive (FP), false-negative (FN), and true-negative (TN) results reported, or data were derivable from the published results. Two authors (H.C.O. and J.Y.L.) examined the titles and abstracts of references identified by the electronic search strategies described above to check the relevance of each study. Studies considered potentially relevant in the search were obtained as full articles and independently assessed for inclusion by the same two authors. Any disagreement was discussed and solved by a third reviewer (J.S.C.).

\section{Data extraction and quality assessment}

Two authors (H.K. and G.J.C.) used a structured, pilot-tested, Excel data collection form to independently extract the data from the included studies. Extracted data included the following study characteristics: (1) name of first author; (2) year of publication; (3) name of journal; (4) study design; (5) number of patients; (6) detection methods; (7) size of core or FNA needle used; (8) absolute numbers in TP, FP, FN, and TN arms, or any data from which this information was derivable; (9) existence of technical failure, overall complication, pancreatitis, and bleeding; (10) needle size; and (11) location from where the sample was obtained. Two authors (H.K. and G.J.C.) also independently assessed methodological quality using the Quality Assessment of Diagnostic Accuracy Studies 2 (QUADAS-2) tool [11]. Any disagreement was discussed and solved by a third reviewer (J.S.C.).

\section{Data analysis}

To evaluate the diagnostic performance, we constructed $2 \times 2$ tables. The TP, FP, TN, and FN values were analyzed to calculate sensitivity and specificity. The Mantel-Haenszel method of the random-effect model was used to calculate pooled sensitivity and specificity with corresponding 95\% confidence intervals (CI), and pooled diagnostic odds ratio (DOR) using the DerSimonian-Laird method [12].

The formula for a positive likelihood ratio (LR) is sen- 
sitivity / (1-specificity), and the formula for a negative LR is (1-sensitivity) / specificity. A DOR can be calculated as the ratio of the odds of positivity in a disease state relative to the odds of positivity in the nondisease state. The value of the DOR ranges from zero to infinity, with higher values indicative of better discriminative performance. A value of 1 indicates that the test does not discriminate between people with and without the disease/ condition [13].

We used summary receiver operating characteristic (SROC) curves to estimate the area under the curve (AUC). The closer the value of the AUC is to 1, the better validated the diagnostic test is. Furthermore, we used a $Q^{*}$ point from the SROC curve to obtain the maximal joint sensitivity and specificity. The $Q^{\star}$ point is the intersection between a symmetrical SROC curve and the antidiagonal line, at which sensitivity equals specificity. Comparing this with other parameters, $Q^{*}$ point is a single-number summarizing the test performance and has the advantage of being less affected by heterogeneity $[14,15]$.

For existence of technical failure, overall complication, pancreatitis, and bleeding we calculated pooled risk ratio (RR) and $95 \%$ CI. If the $95 \%$ CI included a value of 1 , we considered the difference not to be statistically significant. We used the chi-square test and the I-squared test for heterogeneity. A level of $10 \%$ significance $(p<$ $0.10)$ for the chi-square statistic or an $I^{2}$ greater than 50\% was considered to indicate considerable heterogeneity. The Mantel-Haenszel random-effect model was used for these studies, whereas the Mantel-Haenszel fixed model was used for studies that did not demonstrate significant heterogeneity [16]. If substantial heterogeneity was found, the meta-regression technique was used to explore the reasons for the heterogeneity. Meta-regression was performed using a generalization of Littenberg and Moses Linear models. The model was weighted by the inverse of the variance or study size [15].

We also conducted sensitivity analyses to evaluate the influence of individual studies from analyses with a heterogeneity of $I^{2}$ greater than $50 \%$ of the overall effect estimate, by excluding one such study at a time from the analysis. For data expressed with median and interquartile ranges, we changed to mean and standard deviation via the data extraction method in the Cochrane handbook for systematic reviews of intervention [17].

\section{Publication bias}

Deek's funnel plot asymmetry test was used to examine publication bias. Publication bias was conducted by a regression of InDOR against 1 /root (effective sample size), with $p<0.05$ for the slope coefficient indicating significant asymmetry [18].

Two-sided $p<0.05$ was considered statistically significant. Analyses were performed using Meta-DiSc version 1.4 (Unit of Clinical Biostatistics, the Ramón y Cajal Hospital, Madrid, Spain) and Stata 14.0 (Stata Corp., College Station, TX, USA) software. We used the MedCalc Statistical Software version 13.0.2 (MedCalc Software, Ostend, Belgium; http://www.medcalc.org) to calculate the $Z$ statistic of the SROC in order to compare the diagnostic accuracy of core and standard needles, with $p<$ 0.05 indicating have statistical significance.

\section{RESULTS}

\section{Study selection}

The search of Ovid MEDLINE, EMBASE, and CENTRAL produced 22 studies, 20 from database searches and two from manual searching. Of these 22 studies, 11 studies were excluded because, after reviewing the title and abstracts, they appeared to be out of the remit of this study. Eleven studies were included in the final analysis. The literature search process is shown in Fig. 1.

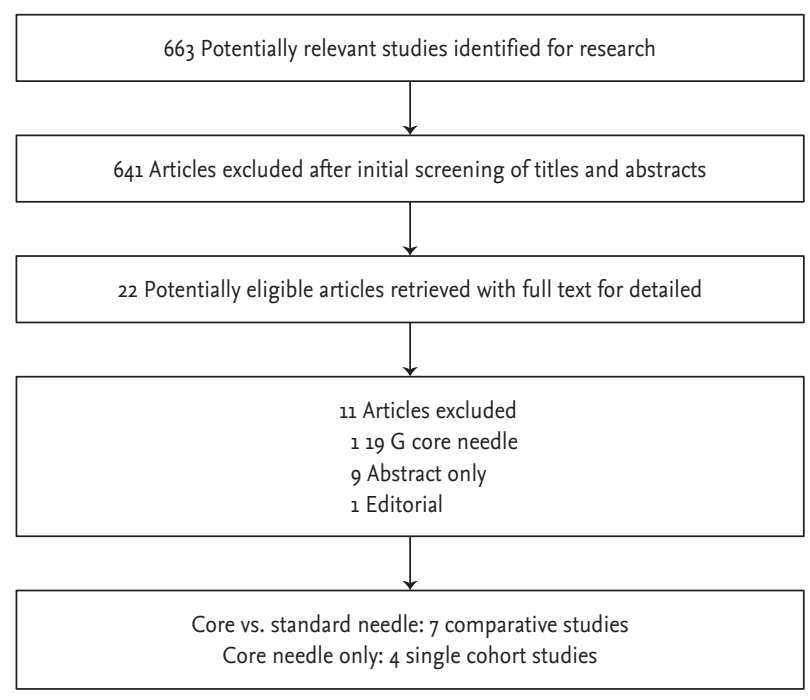

Figure 1. Flow chart of literature search and selection. 


\section{Characteristics of included studies}

Eleven studies involving 896 subjects met the defined inclusion criteria. Of the 896 patients, 792 were in the core needle group and 422 were in the standard needle group (both needles were used in 318 patients). The characteristics of the studies are summarized in Table 1. Seven studies were prospective [7,19-24], four were retrospective [25-28], and four were cross-over studies (three prospective cross-over [20,21,23] and one retrospective cross-over [26]). Seven studies only included the results from pancreatic biopsy [7,19,21-23,27,28], and four studies included overall results (including the pancreas) [20,24-26]. Seven studies compared the core needle with the standard needle [19-23,25,26], and four studies reported only the outcomes of core needle $[7,24,27,28]$.

\section{Quality of included studies}

The quality was moderate in 11 studies, according to the QUADAS-2 items; the results for the distribution of the study design are shown in Appendix 1.

\section{Diagnostic accuracy for all tissue}

For the core needle, the pooled sensitivity and specificity of the diagnosis of malignancy were 0.88 (95\% CI, o.84 to $0.90 ; I^{2}=81.7$, pchi < 0.001 ) and 0.99 (95\% CI, 0.96 to 1.00; $I^{2}=0.0$, pchi $=0.651$ ), respectively. The pooled LR+ and LR- were 16.32 (95\% CI, 7.29 to 36.54; $I^{2}=0.0$, pchi = 0.785 ) and 0.13 (95\% CI, 0.08 to $0.22 ; I^{2}=78.1$, pchi< 0.001 ), respectively. The DOR was 167.37 (95\% CI, 65.77 to 425.91 ; $I^{2}=0.0$, pchi $=0.903$ ) (Fig. 2).

Sensitivity and LR- showed considerable heterogeneity. In the study of Berzosa et al. [26], sensitivity was low

Table 1. Summarized outcomes of studies included in the analysis

\begin{tabular}{|c|c|c|c|c|c|c|c|c|}
\hline \multirow{2}{*}{ Study } & \multirow{2}{*}{ Study design } & \multirow{2}{*}{ Type of needle } & \multirow{2}{*}{$\begin{array}{c}\text { Patients } \\
\text { included, } \\
\text { core vs. } \\
\text { standard }\end{array}$} & \multirow{2}{*}{$\begin{array}{l}\text { Target } \\
\text { organ }\end{array}$} & \multirow{2}{*}{$\begin{array}{l}\text { Mean no. } \\
\text { of pass for } \\
\text { D or A }\end{array}$} & \multicolumn{2}{|c|}{$\begin{array}{c}\text { Procurement, } \\
\text { histologic/optimal core, } \mathrm{n}(\%)\end{array}$} & \multirow{2}{*}{$\begin{array}{c}\text { On-site } \\
\text { pathologist }\end{array}$} \\
\hline & & & & & & $\begin{array}{c}\text { Core } \\
\text { needle }\end{array}$ & $\begin{array}{l}\text { Standard } \\
\text { needle }\end{array}$ & \\
\hline $\begin{array}{l}\text { Bang et al. } \\
(2012)[19]\end{array}$ & $\begin{array}{l}\text { Prospective } \\
\text { randomized }\end{array}$ & $\begin{array}{l}22 \text { G core vs. } \\
\text { standard }\end{array}$ & $28 / 28$ & Pancreas & $1.28 / 1.61(\mathrm{D})$ & $\begin{array}{l}23 / 28(82) \\
22 / 28(80)\end{array}$ & $\begin{array}{l}28 / 28(100) \\
19 / 28(67)\end{array}$ & Yes \\
\hline $\begin{array}{l}\text { Witt et al. } \\
(2013)[25]\end{array}$ & $\begin{array}{l}\text { Retrospective } \\
\text { cohort }\end{array}$ & $\begin{array}{l}22 \text { G core vs. } \\
\text { standard }\end{array}$ & $18 / 18$ & $\begin{array}{l}\text { Pancreas } \\
\text { Lymph nodes } \\
\text { Stomach }\end{array}$ & $2.11 / 2.94(\mathrm{~A})$ & NA & NA & No \\
\hline $\begin{array}{l}\text { Hucl et al. } \\
(2013)[20]\end{array}$ & $\begin{array}{l}\text { Prospective } \\
\text { crossover }\end{array}$ & $\begin{array}{l}22 \text { G core \& } \\
\text { standard }\end{array}$ & $145 / 145$ & $\begin{array}{l}\text { Pancreas } \\
\text { Lymph nodes }\end{array}$ & $1.23 / 2.47(\mathrm{~A})$ & $\begin{array}{r}125 / 145(86) \\
100 / 145(69)\end{array}$ & $\begin{array}{r}127 / 145(88) \\
96 / 145(66)\end{array}$ & No \\
\hline Strand et al. & $\begin{array}{l}\text { Prospective } \\
\text { crossover }\end{array}$ & $\begin{array}{l}22 \text { G core \& } \\
\text { standard }\end{array}$ & $32 / 32$ & Pancreas & $1.4 / 2.9^{\mathrm{a}}$ & $\begin{array}{l}27 / 32(84) \\
19 / 27(70)\end{array}$ & NA & Yes \\
\hline $\begin{array}{l}\text { Lee et al. } \\
(2014)[22]\end{array}$ & $\begin{array}{l}\text { Prospective } \\
\text { randomized }\end{array}$ & $\begin{array}{l}\text { 22/25 G core } \\
\text { vs. standard }\end{array}$ & $58 / 58$ & Pancreas & $1.0 / 2.0(\mathrm{D})^{\mathrm{b}}$ & NA & NA & Yes \\
\hline $\begin{array}{l}\text { Vanbiervliet } \\
\text { et al. (2014) } \\
{[23]}\end{array}$ & $\begin{array}{l}\text { Prospective } \\
\text { crossover }\end{array}$ & $\begin{array}{l}22 \text { G core \& } \\
\text { standard }\end{array}$ & $40 / 40$ & Pancreas & NA & $\begin{array}{l}56 / 80(70) \\
53 / 80(66)\end{array}$ & $\begin{array}{l}70 / 80(88) \\
66 / 80(83)\end{array}$ & No \\
\hline $\begin{array}{l}\text { Berzosa et } \\
\text { al. }(2015)[26]\end{array}$ & $\begin{array}{l}\text { Retrospective } \\
\text { cohort }\end{array}$ & $\begin{array}{l}22 \text { G core \& } 25 \\
\text { G standard }\end{array}$ & $61 / 61$ & Pancreas & $1.7 / 3.5$ & NA & NA & $\begin{array}{c}\text { Yes } \\
(1 / 2 \text { centers })\end{array}$ \\
\hline $\begin{array}{l}\text { Iwashita et } \\
\text { al. }(2013)[27]\end{array}$ & $\begin{array}{l}\text { Retrospective } \\
\text { single arm }\end{array}$ & 25 G core & 50 & Pancreas & $4^{b}$ & $\begin{array}{c}\text { NA } \\
16 / 50(32)\end{array}$ & - & No \\
\hline $\begin{array}{l}\text { Larghi et al. } \\
(2013)[7]\end{array}$ & $\begin{array}{l}\text { Prospective } \\
\text { single arm }\end{array}$ & $22 \mathrm{G}$ core & 61 & Pancreas & NA & $\begin{array}{l}55 / 60(92) \\
54 / 60(89)\end{array}$ & - & No \\
\hline $\begin{array}{l}\text { Fabbri et al. } \\
(2015)[28]\end{array}$ & $\begin{array}{l}\text { Retrospective } \\
\text { single arm }\end{array}$ & $22 \mathrm{G}$ core & 68 & Pancreas & $1.5^{\mathrm{a}}$ & $\begin{array}{l}58 / 68(85) \\
36 / 68(53)\end{array}$ & - & No \\
\hline $\begin{array}{l}\text { Paik et al. } \\
(2015)[24]\end{array}$ & $\begin{array}{c}\text { Prospective } \\
\text { single arm }\end{array}$ & $22 \mathrm{G}$ core & 133 & $\begin{array}{l}\text { Pancreas } \\
\text { Lymph nodes } \\
\text { Others }\end{array}$ & NA & NA & - & No \\
\hline
\end{tabular}

D, diagnosis; A, adequacy; G, gauge; NA, not available.

${ }^{\mathrm{a}}$ Mean number of passes per lesion irrespective of diagnosis and adequacy.

${ }^{\mathrm{b}}$ Median number of passes. 


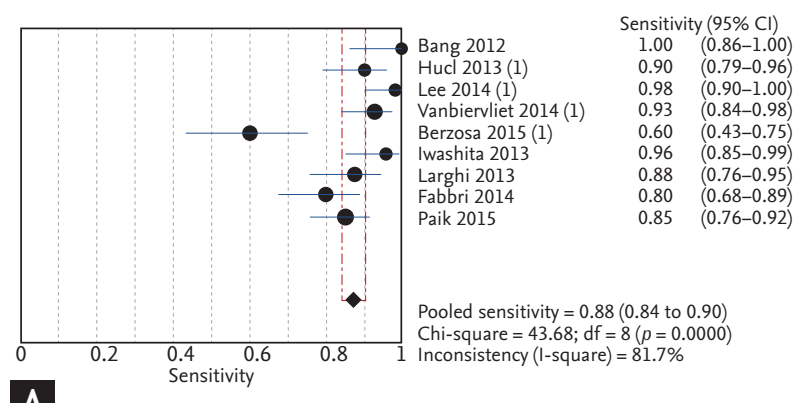

A

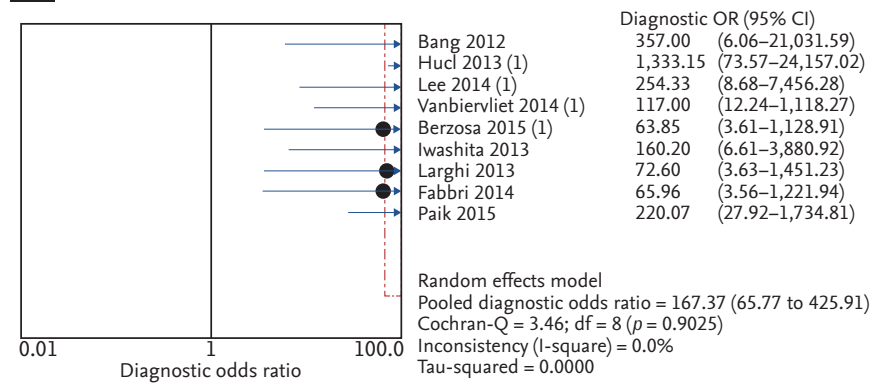

C

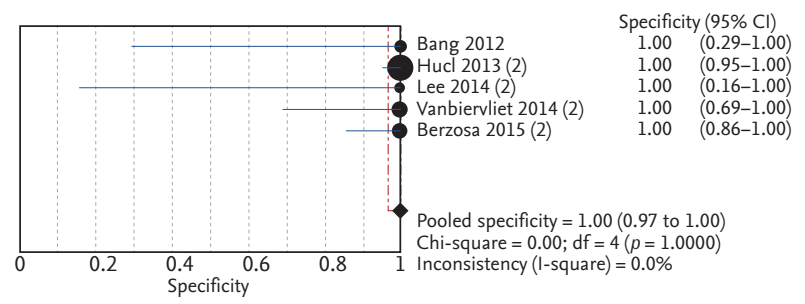

E
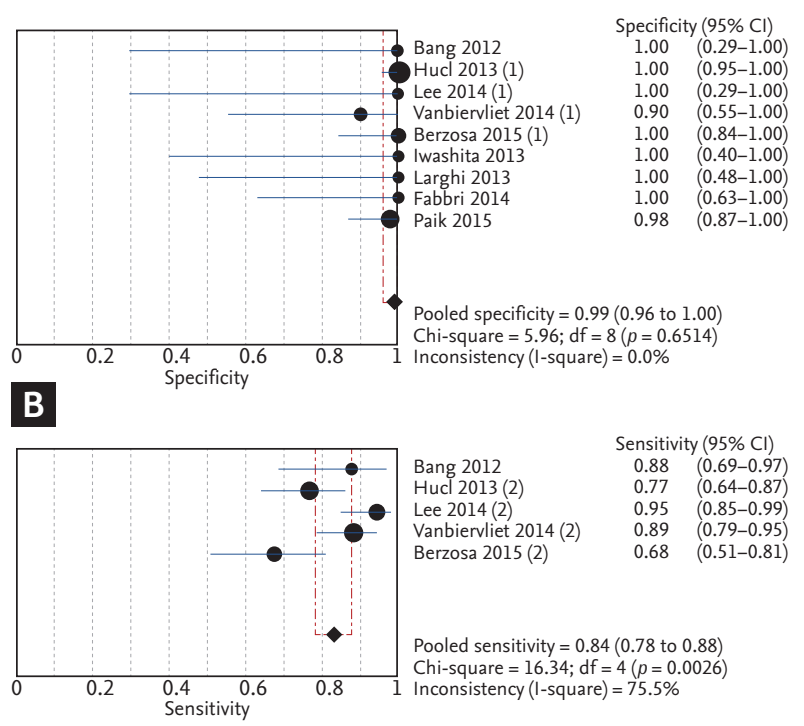

D

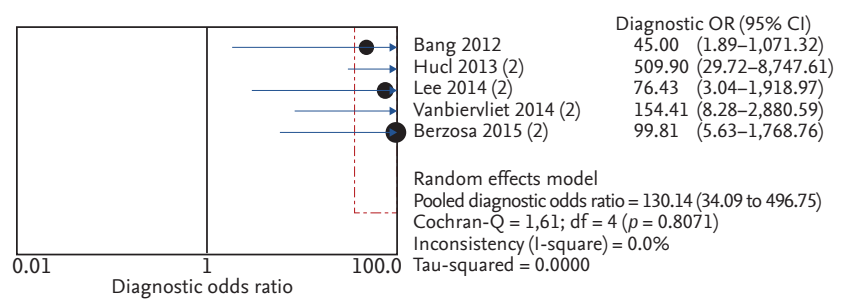

$\mathbf{F}$

Figure 2. Sensitivity, specificity, and diagnostic odd ratios of (A, B, C) the core and (D, E, F) standard needles for the diagnosis of malignancy. CI, confidence interval; OR, odds ratio.

compared with other studies. To exclude the effect of the results of Berzosa et al. [26], a sensitivity analysis was performed. After excluding the study of Berzosa et al. [26], sensitivity increased and heterogeneity decreased; however, it was still substantial; 0.90 (95\% CI, 0.87 to $0.92 ; I^{2}=68.2$, pchi $=0.0026$ ). Both pooled LR- and heterogeneity decreased (0.12; 95\% CI, 0.08 to $0.17 ; I^{2}=43.9$, pchi $=0.0859$ ).

For the standard needle, pooled sensitivity and specificity for the diagnosis of malignancy were 0.84 ( $95 \% \mathrm{CI}$, 0.79 to $\left.0.88 ; I^{2}=75.5, p c h i=0.003\right)$ and $1.00(95 \% \mathrm{CI}, 0.97$ to $1.00 ; I^{2}=0.0$, pchi $=1.000$ ), respectively. Pooled LR+ and LR- were 18.49 (95\% CI, 5.61 to 60.95; $\mathrm{I}^{2}=0.0$, pchi $=0.4384$ ) and 0.19 (95\% CI, 0.12 to $0.30 ; I^{2}=63.2$, pchi $=$ 0.028), respectively. The DOR was 130.14 (95\% CI, 34.09 to $496.75 ; I^{2}=0.0$, pchi $=0.8071$ ) (Fig. 2 ).

The AUC of the core and standard needles in the diagnosis of malignancy was 0.974 (standard error [SE] [AUC]
$=0.011$ ) and $0.955(\mathrm{SE}[\mathrm{AUC}]=0.029)$, respectively (Fig. 3). The Z statistic compared with these two SROC was 0.613 ( $p=0.540)$.

A substantial degree of heterogeneity was observed in the sensitivity and pooled LR- for both groups. Since obvious heterogeneity was observed, the meta-regression technique was used to explore heterogeneity other than threshold effect; there were no significant factors (Table 2).

\section{Diagnostic accuracy for pancreatic tissue}

For core needle, the pooled sensitivity and specificity for the diagnosis of pancreatic malignancy were 0.91 (95\% CI, 0.88 to $\left.0.94 ; I^{2}=73.7, p c h i=0.002\right)$ and $0.97(95 \%$ CI, 0.84 to $1.00 ; I^{2}=0.0$, pchi $=0.783$ ), respectively. Pooled LR+ and LR- were 9.54 (95\% CI, 3.53 to 25.81; $I^{2}=0.0$, pchi $=1.000$ ) and 0.10 (95\% CI, 0.05 to $0.18 ; I^{2}=59.6$, pchi = 0.030), respectively. The DOR was 125.24 (95\% CI, 36.95 to 

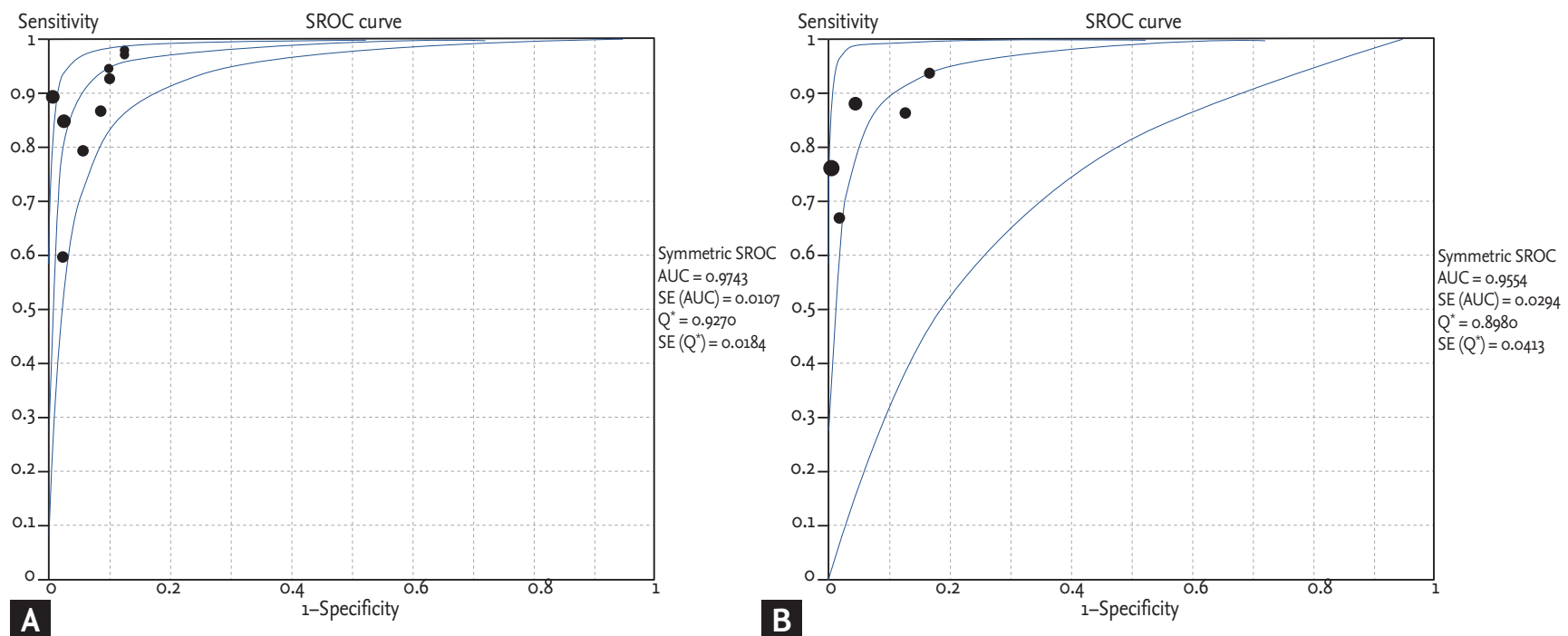

Figure 3. Summary receiver operating characteristic (SROC) curves of (A) the core and (B) standard needles in the diagnosis of malignancy. AUC, area under the curve; SE, standard error.

Table 2. Meta-regression of potential sources of heterogeneity

\begin{tabular}{|c|c|c|c|c|c|}
\hline Variance & Coefficient standard & Standard error & $p$ value & RDOR & $95 \% \mathrm{CI}$ \\
\hline Cte & 3.493 & 2.4058 & 0.1771 & NA & NA \\
\hline S & 0.193 & 0.4481 & 0.6764 & NA & NA \\
\hline Method $^{\mathrm{a}}$ & -0.152 & 0.8782 & 0.8656 & 0.86 & $0.12-6.08$ \\
\hline Tissue & 1.382 & 1.5709 & 0.3998 & 3.98 & $0.12-131.87$ \\
\hline
\end{tabular}

The RDOR means the diagnostic odds ratio (DOR) for studies that lacked a particular methodological feature divided by the DOR for studies without the flaw. $p<0.05$ indicates a statistically significant relationship between the characteristics of studies and the DOR.

RDOR, relative diagnostic odds ratio; CI, confidence interval; Cte, constant term in the equation; NA, not available; S, indicator of threshold.

${ }^{\mathrm{a}}$ Method means the type of needle.

424.41; $I^{2}=0.0$, pchi = 0.976) (Fig. 4).

In the study of Berzosa et al. [26], the sensitivity was too low. To exclude the effect of the results of this study, a sensitivity analysis was performed. Sensitivity increased and heterogeneity decreased; however, this still remained substantial (0.91; 95\% CI, 0.88 to $0.94 ; I^{2}$ $=73.7$, pchi $=0.0019)$. The heterogeneity of pooled LRdecreased, but was also still substantial (95\% CI, 0.05 to o.18; $I^{2}=59.6$, pchi = 0.030).

For the standard needle, the pooled sensitivity and specificity for the diagnosis of pancreatic malignancy were 0.91 (95\% CI, 0.85 to $0.95 ; I^{2}=0.0$, pchi $\left.=0.416\right)$ and 1.00 (95\% CI, 0.78 to $1.00 ; I^{2}=0.0$, pchi $=1.000$ ), respectively. Pooled LR+ and LR- were 8.84 (95\% CI, 1.96 to 39.90; $I^{2}=0.0$, pchi = 0.769) and 0.12 (95\% CI, o.07 to 0.19;
$I^{2}=0.0$, pchi $\left.=0.618\right)$, respectively. The DOR was 83.97 (95\% CI, 14.04 to 502.29; $I^{2}=0.0$, pchi = 0.848) (Fig. 4).

The AUC values of the core and standard needles in the diagnosis of pancreatic malignancy were 0.966 (SE $[\mathrm{AUC}]=0.017$ ) and 0.957 (SE $[\mathrm{AUC}]=0.029)$, respectively (Fig. 5). The $Z$ statistic compared with these two SROC was $0.238(p=0.789)$.

\section{Histologic core, adequacy, and number of passes for diagnosis}

The procurement of a histologic core with the core needle was evaluated in four studies $[7,19,23,28]$. The probability for obtaining a histologic core was 0.828 (95\% CI, 0.707 to $0.906 ; I^{2}=72.26 \%$, pchi $\left.=0.013\right)$. The procurement of histologic cores with the core needle was compared 


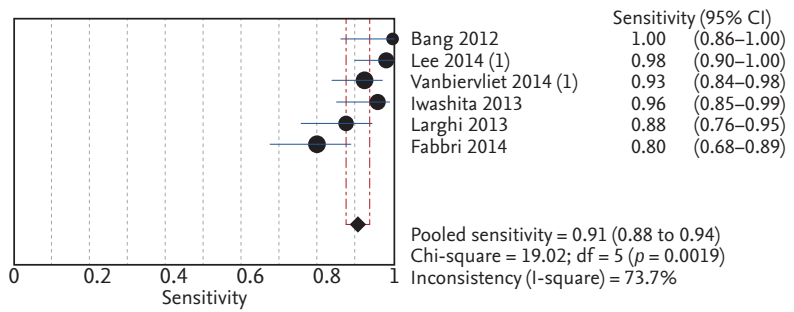

A

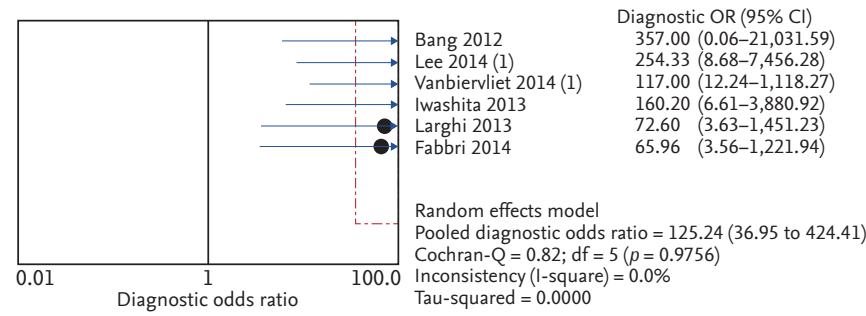

C

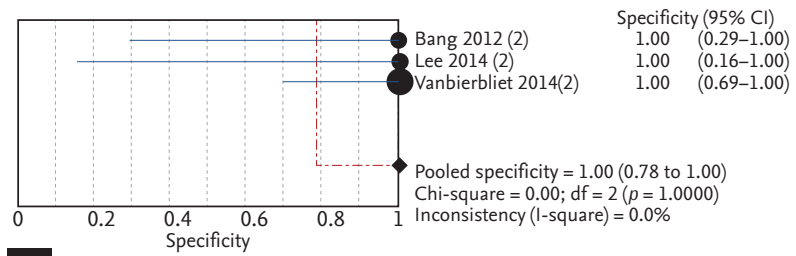

E

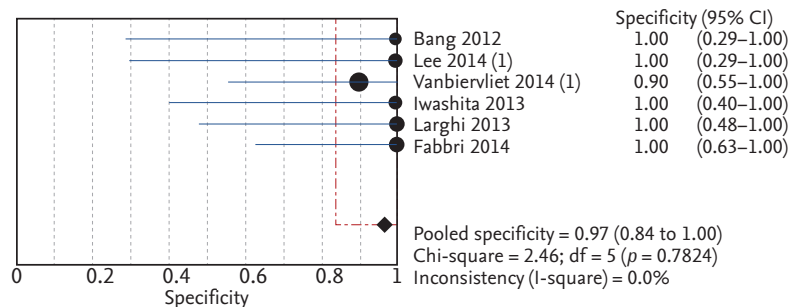

B

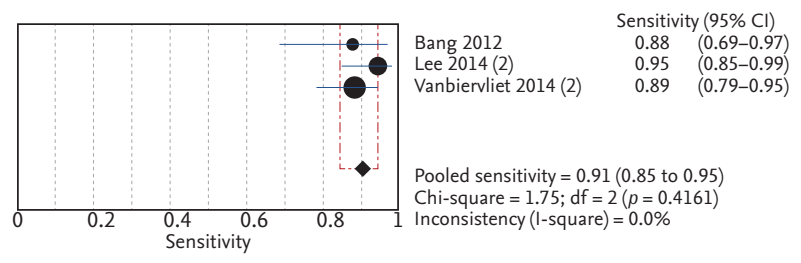

D

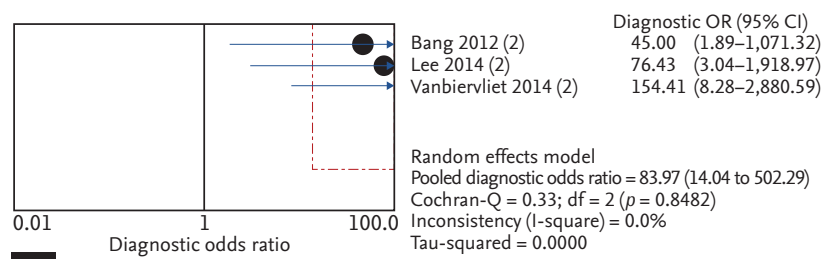

$\mathbf{F}$

Figure 4. Sensitivity, specificity, and diagnostic odd ratio of (A, B, C) the core and (D, E, F) standard needles for the diagnosis of pancreatic malignancy. $\mathrm{CI}$, confidence interval; OR, odds ratio.

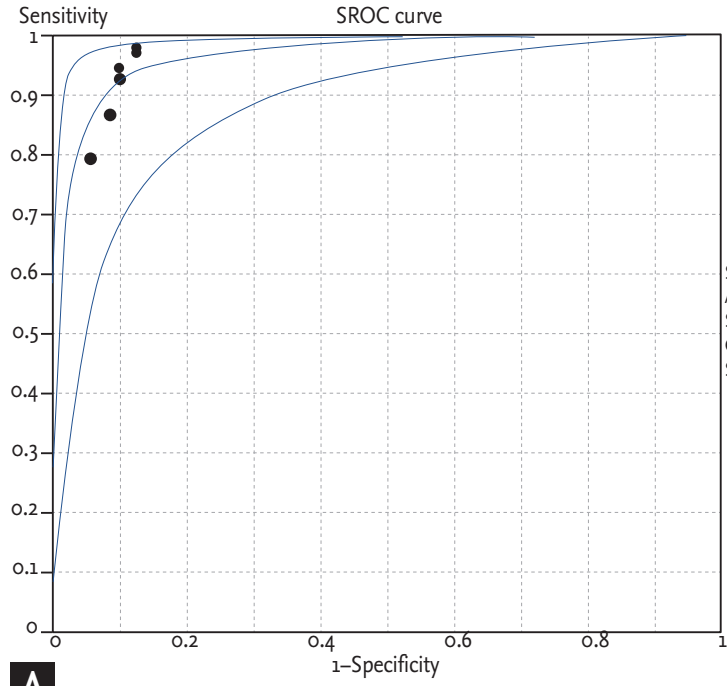

A

Figure 5 Summary receiver operating characteristic (SROC) curves of(A) the core and (B) standard needles in the diagnosis of pancreatic malignancy. AUC, area under the curve; SE, standard error.

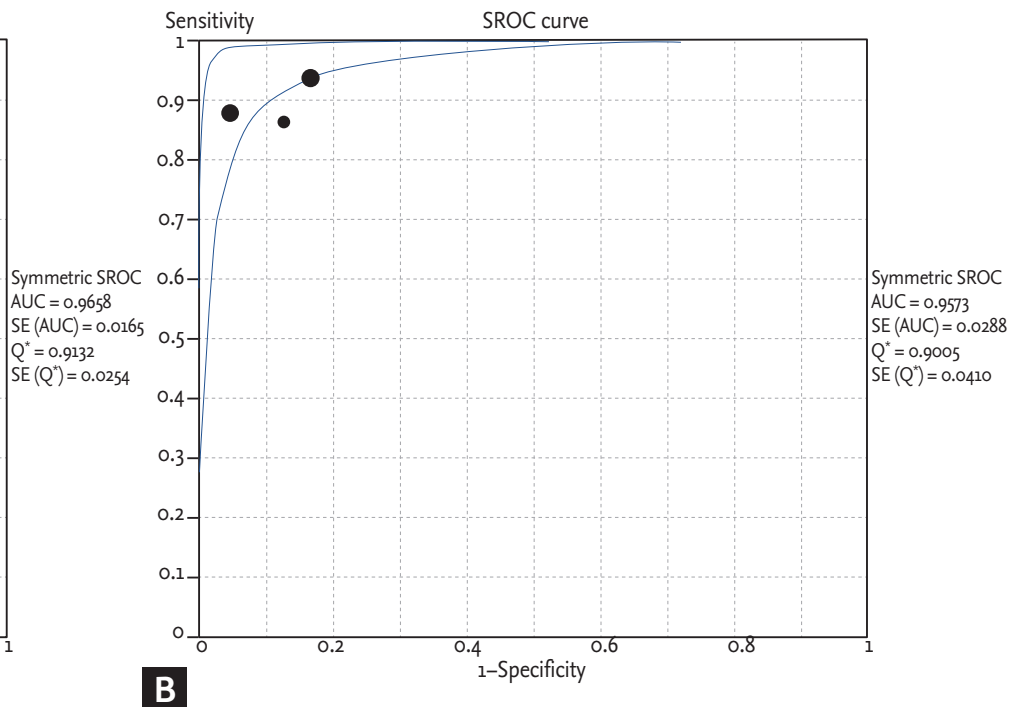


with that of the standard needle in two studies [19,23]. Compared with the standard needle, the histologic core procurement of the core needle was significantly lower (RR, 0.81; 95\% CI, 0.72 to $0.92 ; I^{2}=0 \%$, pchi = 0.810).

The procurement of an optimal core with the core needle was evaluated in seven studies [7,19-21,23,27,28]. The probability of obtaining an optimal core was 0.666 (95\% CI, 0.530 to $0.779 ; I^{2}=85.59 \%$, pchi < 0.000 ). The procurement of an optimal core with the core needle was compared with that of the standard needle in three studies $[19,20,23]$. The combined results showed no evidence for a statistical difference (RR, 0.545; 95\% CI, 0.187 to $1.589 ; I^{2}=74.68 \%$, pchi $=0.019$ ).

Adequacy of sample with the core needle was compared with that of the standard needle in four studies $[20,23,25,26]$. The combined results indicated that there was no statistical difference (RR, 0.96; $95 \% \mathrm{CI}, 0.90$ to 1.02; $I^{2}=0 \%$, pchi $=0.837$ ).

The number of passes for diagnosis with the core needle was compared with that of the standard needle in two studies $[19,22]$. It was significantly lower with the core needle compared with the standard needle (standardized mean difference $-0.72 ; 95 \% \mathrm{CI},-1.02$ to $-0.41 ; \mathrm{I}^{2}$ $=30.0 \%$, pchi $=0.232$ ).

\section{Technical failure}

Technical failure with the core needle was compared with that of the standard needle in four studies [19,2123]. The combined results showed no statistical difference (RR, 5.07; 95\% CI, 0.68 to 37.63; $\mathrm{I}^{2}=0 \%$, pchi $\left.=0.864\right)$.

Overall complications with the core needle were compared with that of the standard needle in six studies $[19,20,22,23,25,26]$. The combined results showed no evidence of a statistical difference (RR, 1.26; $95 \%$ CI, 0.34 to $4.62 ; \mathrm{I}^{2}=0 \%$, pchi $\left.=0.878\right)$. Pancreatitis and bleeding with core needle were compared with that of the standard needle in five $[19,20,22,25,26]$ and two studies [22,23], respectively. The combined results did not show a statistical difference (RR, 1.35; 95\% CI, 0.26 to $7.10 ; I^{2}=0 \%$, pchi $=0.987)$ and (RR, 1.30; 95\% CI, 0.15 to $11.05 ; I^{2}=53.17 \%$, pchi $=0.144$, respectively).

\section{Publication bias}

The $p$ value for the slope coefficient in Deek's funnel plot asymmetry test was 0.797 , suggesting a low likelihood of publication bias (Appendix 2).

\section{DISCUSSION}

This systematic review and meta-analysis included a large cohort of patients $(n=896)$ and quantitatively summarized the available evidence regarding the outcomes with the core needle in comparison with the standard needle. This study demonstrated that the diagnostic outcomes, including accuracy for diagnosis of malignancy and adequacy of sample, were comparable between core and standard needles and there was no significant difference in procurement of optimal histologic cores between the core and standard needles.

The anticipated advantage of the core needle is the obtainment of core tissue for histopathologic diagnosis and an increase in the diagnostic accuracy. However, the procurement of core tissue was significantly lower with the core needle compared with the standard needle. There was no significant difference in the procurement of optimal cores between both needles. The procurement of optimal core tissue appeared to vary according to the needle caliber; $32 \%$ with $25 \mathrm{G}$ core needle [27], $53 \%$ to $89 \%$ with 22 G core needle [7,19-21,23,28], and 90\% with 19 G core needle [6]. In the study, with the lowest rate of $32 \%$, capillary sampling with the stylet slow-pull technique by $25 \mathrm{G}$ core needle was adopted [27]. It was assumed that the small caliber $(25 \mathrm{G})$ of core needle, rather than the application of suction, was associated with the low procurement of core tissue [27]. On the other hand, the presence of tissue trap in the core needle may prevent the full application of capillary suction within the needle. Even though reverse bevel may increase the shearing of tissue from the mass, the sheared tissue may not be efficiently entrapped and piled up within the needle, and this may result in a lower procurement of core tissue. With the standard needle, a small caliber and application of low or no negative suction tends to produce less bloody specimens and improves the cytologic yield [29,30]. However, there was no significant difference in the procurement rate of core tissue between the application of high negative suction and the capillary sampling method with the core needle (85\% and $92 \%[9,10]$ vs. $89 \%[24])$.

The presence of whitish materials in specimens obtained by a 19 G standard needle has been used as an indicator for adequacy of sample, and a high correlation of these visible cores with adequate histologic cores has been reported [31,32]. However, a distinct discrepancy 
between visible cores and histologic cores (92\% vs. 32\%) has been reported with the $25 \mathrm{G}$ core needle; this is assumed to be due to the smaller needle [27].

The optimal number of needle passes with the standard needle to obtain a correct diagnosis of pancreatic lesions has been reported as seven passes, with a sensitivity of $83 \%$ [33]. Core needle sampling allows both cytologic and histologic analysis. Combined diagnostic yield would likely reduce the number of needle passes for diagnosis. In the present study, the number of needle passes for diagnosis was significantly lower with the core needle than the standard needle. The sensitivity for combined cytologic and histologic analysis reached $82 \%$ to $85 \%$ for a single pass and $89 \%$ to $96 \%$ for multiple passes $[24,27]$. A distinctive advantage of the core needle might be the higher cost-effectiveness of performing a single pass, while maintaining sufficient diagnostic accuracy. However, as a single pass is not always sufficient for the correct diagnosis, multiple passes or waiting for interpretation after one pass is required. In addition, there is no clear advantage of one or two passes with core needle over three passes with standard needle. Three passes using a $25 \mathrm{G}$ standard needle is faster, easier and less traumatic than two passes with a large core needle.

Even though the technical success rate of puncturing from the duodenum with the $19 \mathrm{G}$ core needle was relatively high in one study (94\%) [6], the bended endoscope position in the duodenum impairs its proper function. For this lesion, new 22/25 G core needle platforms have been developed and evaluated for their feasibility and diagnostic efficacy. Technical failure of the core needle was described in seven cases from three studies [19,21,27], and occurred in six cases during the transduodenal approach. In the comparison of both needles, there was significant difference in technical failure with the 22/25 $\mathrm{G}$ needle. For the transduodenal approach, the core needle needed to be pushed out of the echoendoscope in the stomach before advancing into the duodenum [6].

One of the important indication for doing core biopsy is to get more tissue for histologic evaluation of architecture. Nowadays, the need for enough sample for genetic testing is increasing. The problem with the published studies is that they do not provide a clear measurement of how much tissue was obtained for which test (for example, genetic testing or counting mitotic figures in gastrointestinal stromal tumor).
There were some limitations in this review. First, there was substantial heterogeneity in the selected studies. Despite reductions in heterogeneity after subgroup and sensitivity analysis, it remained substantial and its cause could not be determined. Variations in basic characteristics of selected studies, including defined criteria for adequacy of sample and histologic cores, and availability of on-site pathologist, may have potentially introduced heterogeneity. Second, to include as many eligible studies as possible and to exclude the language bias in this systematic review, the authors did not apply any language restrictions while searching the database. However, all selected studies were published in English. There may have been some other language publications that are not included in this review. Although publication bias was determined to be low by statistical analysis, the possibility of publication bias may not be fully excluded.

In conclusion, core and standard needles were comparable in terms of diagnostic accuracy, technical performance and safety profile. The number of needle passes for diagnosis was significantly lower with the core needle than the standard needle. However, there was no significant difference in the procurement of optimal histologic cores between both needles. Therefore, if the aim is to confirm a malignancy, either core or standard needles can be used based on endosonographer preference.

\section{KEY MESSAGE}

1. Core and standard needles were comparable in terms of diagnostic accuracy.

2. The number of needle passes for diagnosis was significantly lower with the core needle.

3. There was no significant difference in the procurement of optimal histologic cores between core and standard needles.

\section{Conflict of interest}

No potential conflict of interest relevant to this article was reported.

\section{REFERENCES}

1. Puli SR, Bechtold ML, Buxbaum JL, Eloubeidi MA. How 
good is endoscopic ultrasound-guided fine-needle aspiration in diagnosing the correct etiology for a solid pancreatic mass? A meta-analysis and systematic review. Pancreas 2013;42:20-26.

2. Hewitt MJ, McPhail MJ, Possamai L, Dhar A, Vlavianos P, Monahan KJ. EUS-guided FNA for diagnosis of solid pancreatic neoplasms: a meta-analysis. Gastrointest Endosc 2012;75:319-331.

3. Caraway NP. Evolving role of FNA biopsy in diagnosing lymphoma: past, present, and future. Cancer Cytopathol 2015;123:389-393.

4. DeWitt J, Emerson RE, Sherman S, et al. Endoscopic ultrasound-guided Trucut biopsy of gastrointestinal mesenchymal tumor. Surg Endosc 2011;25:2192-2202.

5. Larghi A, Verna EC, Stavropoulos SN, Rotterdam H, Lightdale CJ, Stevens PD. EUS-guided trucut needle biopsies in patients with solid pancreatic masses: a prospective study. Gastrointest Endosc 2004;59:185-190.

6. Iglesias-Garcia J, Poley JW, Larghi A, et al. Feasibility and yield of a new EUS histology needle: results from a multicenter, pooled, cohort study. Gastrointest Endosc 2011;73:1189-1196.

7. Larghi A, Iglesias-Garcia J, Poley JW, et al. Feasibility and yield of a novel 22-gauge histology EUS needle in patients with pancreatic masses: a multicenter prospective cohort study. Surg Endosc 2013;27:3733-3738.

8. Moher D, Liberati A, Tetzlaff J, Altman DG; PRISMA Group. Preferred reporting items for systematic reviews and meta-analyses: the PRISMA statement. Open Med 2009;3:e123-e130.

9. Stroup DF, Berlin JA, Morton SC, et al. Meta-analysis of observational studies in epidemiology: a proposal for reporting: Meta-analysis Of Observational Studies in Epidemiology (MOOSE) group. JAMA 2000;283:2008-2012.

10. Leeflang MM, Deeks JJ, Gatsonis C, Bossuyt PM; Cochrane Diagnostic Test Accuracy Working Group. Systematic reviews of diagnostic test accuracy. Ann Intern Med 2008;149:889-897.

11. Whiting P, Rutjes AW, Reitsma JB, Bossuyt PM, Kleijnen J. The development of QUADAS: a tool for the quality assessment of studies of diagnostic accuracy included in systematic reviews. BMC Med Res Methodol 2003;3:25.

12. Reitsma JB, Glas AS, Rutjes AW, Scholten RJ, Bossuyt PM, Zwinderman $\mathrm{AH}$. Bivariate analysis of sensitivity and specificity produces informative summary measures in diagnostic reviews. J Clin Epidemiol 2005:58:982-990.
13. Glas AS, Lijmer JG, Prins MH, Bonsel GJ, Bossuyt PM. The diagnostic odds ratio: a single indicator of test performance. J Clin Epidemiol 2003;56:1129-1135.

14. Irwig L, Macaskill P, Glasziou P, Fahey M. Meta-analytic methods for diagnostic test accuracy. J Clin Epidemiol 1995;48:119-130.

15. Moses LE, Shapiro D, Littenberg B. Combining independent studies of a diagnostic test into a summary ROC curve: data-analytic approaches and some additional considerations. Stat Med 1993;12:1293-1316.

16. Higgins JP, Thompson SG, Deeks JJ, Altman DG. Measuring inconsistency in meta-analyses. BMJ 2003;327:557-560.

17. Higgins JP, Green S; Cochrane Collaboration. Cochrane Handbook for Systematic Reviews of Interventions. Oxford: Cochrane Collaboration, 2011.

18. Deeks JJ, Macaskill P, Irwig L. The performance of tests of publication bias and other sample size effects in systematic reviews of diagnostic test accuracy was assessed. J Clin Epidemiol 2005;58:882-893.

19. Bang JY, Hebert-Magee S, Trevino J, Ramesh J, Varadarajulu S. Randomized trial comparing the 22-gauge aspiration and 22-gauge biopsy needles for EUS-guided sampling of solid pancreatic mass lesions. Gastrointest Endosc 2012;76:321-327.

20. Hucl T, Wee E, Anuradha S, et al. Feasibility and efficiency of a new $22 \mathrm{G}$ core needle: a prospective comparison study. Endoscopy 2013;45:792-798.

21. Strand DS, Jeffus SK, Sauer BG, Wang AY, Stelow EB, Shami VM. EUS-guided 22-gauge fine-needle aspiration versus core biopsy needle in the evaluation of solid pancreatic neoplasms. Diagn Cytopathol 2014;42:751-758.

22. Lee YN, Moon JH, Kim HK, et al. Core biopsy needle versus standard aspiration needle for endoscopic ultrasound-guided sampling of solid pancreatic masses: a randomized parallel-group study. Endoscopy 2014;46:10561062.

23. Vanbiervliet G, Napoleon B, Saint Paul MC, et al. Core needle versus standard needle for endoscopic ultrasound-guided biopsy of solid pancreatic masses: a randomized crossover study. Endoscopy 2014;46:1063-1070.

24. Paik WH, Park Y, Park do H, et al. Prospective evaluation of new 22 gauge endoscopic ultrasound core needle using capillary sampling with stylet slow-pull technique for intra-abdominal solid masses. J Clin Gastroenterol 2015;49:199-205.

25. Witt BL, Adler DG, Hilden K, Layfield LJ. A comparative 
needle study: EUS-FNA procedures using the HD ProCor and EchoTip 22-gauge needle types. Diagn Cytopathol 2013;41:1069-1074.

26. Berzosa M, Villa N, El-Serag HB, Sejpal DV, Patel KK. Comparison of endoscopic ultrasound guided 22-gauge core needle with standard 25-gauge fine-needle aspiration for diagnosing solid pancreatic lesions. Endosc U1trasound 2015;4:28-33.

27. Iwashita T, Nakai Y, Samarasena JB, et al. High single-pass diagnostic yield of a new 25-gauge core biopsy needle for EUS-guided FNA biopsy in solid pancreatic lesions. Gastrointest Endosc 2013;77:909-915.

28. Fabbri C, Luigiano C, Maimone A, et al. Endoscopic ultrasound-guided fine-needle biopsy of small solid pancreatic lesions using a 22-gauge needle with side fenestration. Surg Endosc 2015;29:1586-1590.

29. Siddiqui UD, Rossi F, Rosenthal LS, Padda MS, Murali-Dharan V, Aslanian HR. EUS-guided FNA of solid pancreatic masses: a prospective, randomized trial compar- ing 22-gauge and 25-gauge needles. Gastrointest Endosc 2009;70:1093-1097.

30. Yusuf TE, Ho S, Pavey DA, Michael H, Gress FG. Retrospective analysis of the utility of endoscopic ultrasound-guided fine-needle aspiration (EUS-FNA) in pancreatic masses, using a 22-gauge or 25-gauge needle system: a multicenter experience. Endoscopy 2009;41:445-448.

31. Yasuda I, Goto N, Tsurumi H, et al. Endoscopic ultrasound-guided fine needle aspiration biopsy for diagnosis of lymphoproliferative disorders: feasibility of immunohistological, flow cytometric, and cytogenetic assessments. Am J Gastroenterol 2012;107:397-404.

32. Iwashita T, Yasuda I, Doi S, et al. Use of samples from endoscopic ultrasound-guided 19-gauge fine-needle aspiration in diagnosis of autoimmune pancreatitis. Clin Gastroenterol Hepatol 2012;10:316-322.

33. LeBlanc JK, Ciaccia D, Al-Assi MT, et al. Optimal number of EUS-guided fine needle passes needed to obtain a correct diagnosis. Gastrointest Endosc 2004;59:475-481. 


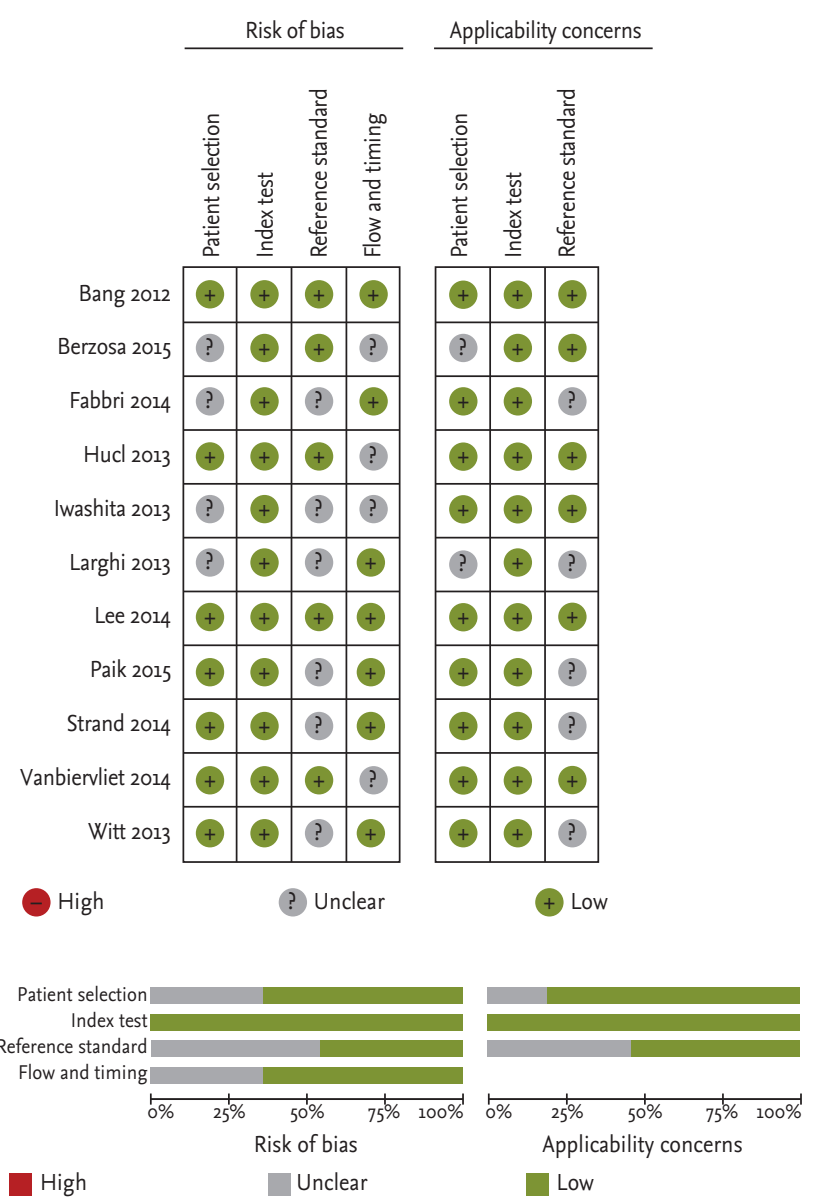

Appendix 1. Methodological quality of the 11 included studies. (A) Methodological quality graph: each methodological quality item is presented as the percentages across all included studies. (B) Methodological quality summary. 
Oh HC, et al. Core needle in EUS-guided sampling

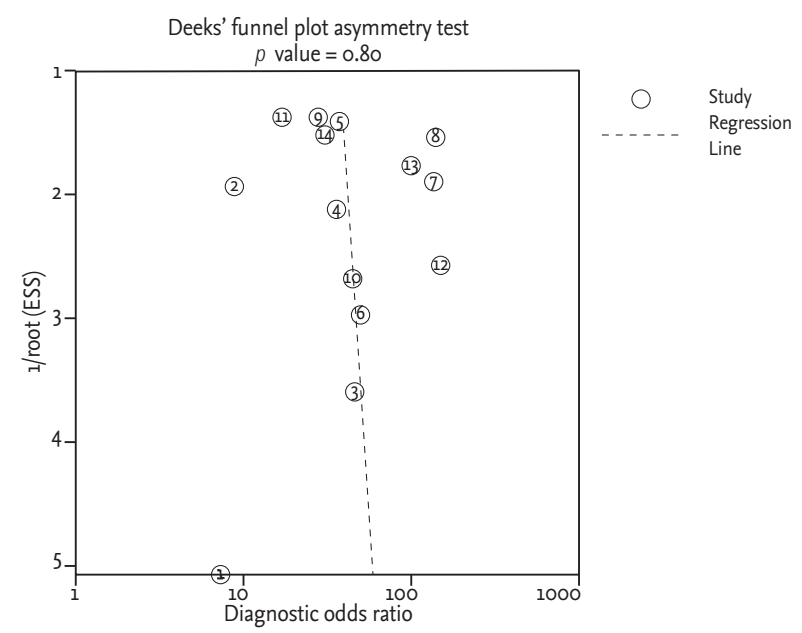

Appendix 2. Publication bias. ESS, effective sample size. 